\title{
Order parameter in superconductors with non-degenerate bands
}

\author{
I. A. Sergienkd $*$ and S. H. Curnoe \\ Department of Physics and Physical Oceanography, Memorial University of Newfoundland, \\ St. John's, Newfoundland E Labrador A1B 3X\%, Canada
}

\begin{abstract}
In noncentrosymmetric metals, the spin degeneracy of the electronic bands is lifted by spin-orbit coupling. We consider general symmetry properties of the pairing function $\Delta(\mathbf{k})$ in noncentrosymmetric superconductors with spin-orbit coupling (NSC), including $\mathrm{CePt}_{3} \mathrm{Si}$, UIr and $\mathrm{Cd}_{2} \mathrm{Re}_{2} \mathrm{O}_{7}$. We find that $\Delta(\mathbf{k})=\chi(\mathbf{k}) t(\mathbf{k})$, where $\chi(\mathbf{k})$ is an even function which transforms according to the irreducible representations of the crystallographic point group and $t(\mathbf{k})$ is a model dependent phase factor. We consider tunneling between a NSC and a conventional superconductor. It is found that, in terms of thermodynamical properties as well as the Josephson effect, the state of NSC resembles a singlet superconducting state with gap function $\chi(\mathbf{k})$.

PACS numbers: 74.20.-z, 74.20.Rp, 74.50.+r, 71.27.+a
\end{abstract}

\section{INTRODUCTION}

A better understanding of unconventional superconductivity has been sought ever since the pioneering discovery of heavy fermion superconductivity in $\mathrm{CeCu}_{2} \mathrm{Si}_{2}$ Soon after, the effect of spin-orbit coupling (SOC) was recognized to play an important role in the superconducting (SC) properties of heavy fermion materials, because it breaks spin rotation symmetry. ${ }^{2.3}$ If there is no spin-orbit coupling, or if the crystal has space inversion and time reversal symmetry, the electronic bands are doubly degenerate everywhere in the Brillouin zone. $\stackrel{4}{\underline{4}}$ Then, the superconducting states can be classified as spin-singlet (even parity) and spin-triplet (odd parity), where the spin is replaced by pseudospin when SOC is present 2,5.6.7 However, if inversion symmetry is absent and SOC is present, the degeneracy of electronic band states is removed at all points in the Brillouin zone except at some highly symmetric positions, while time reversal symmetry ensures that states with opposite momenta are degenerate. 4

The recent interest in noncentrosymmetric superconductors was stimulated by the discovery of superconductivity in $\mathrm{CePt}_{3} \mathrm{Si}$ and $\mathrm{UIr}{ }^{8.9} \mathrm{SOC}$ is normally strong in cerium and uranium compounds since the atoms are heavy. A recent band structure calculation of $\mathrm{CePt}_{3} \mathrm{Si}$ found that the bands, which would be degenerate if SOC was absent, are split by $50-200 \mathrm{meV}$ for the states close to Fermi level ${ }^{10}$ This splitting energy is a factor of more than a thousand on the characteristic energy scale $k_{B} T_{c}$. Therefore, the one-band theory, which applies to the spin-degenerate case, should be reformulated 11

Actually, several superconducting materials in which inversion symmetry is absent have been known for some time. Sesquicarbide materials, with chemical formula $\mathrm{R}_{2} \mathrm{C}_{3-y}$ ( $\mathrm{R}=\mathrm{La}$ or $\mathrm{Y}$, which can be partially substituted by a number of elements) have $T_{c}$ 's of up to 18 $\mathrm{K}$, and space group symmetry $I \overline{4} 3 d d^{12,13,14}$ This belongs to the tetrahedral crystallographic class $T_{d}$ which has no symmetry centre. However, in sesquicarbides SOC does not seem to be important for conduction electrons, i.e. the bands are still spin degenerate.15,16
In 2001, superconductivity with $T_{c}=1 \mathrm{~K}$ was reported in $\mathrm{Cd}_{2} \mathrm{Re}_{2} \mathrm{O}_{7} \cdot 17,18$ At room temperature, this material has the ideal pyrochlore structure, which includes a centre of symmetry. However, superconductivity occurs on the background of a noncentrosymmetric tetragonal crystal field after a series of structural phase transitions, ${ }^{19,20,21}$ In addition, SOC dramatically affects the electronic band structure, as shown by first-principles calculations for the pyrochlore phase ${ }^{22.23}$ Following further calculations, ${ }^{24}$ it was predicted that if the centre of symmetry is removed from the structure, the spin-orbit splitting of the bands reaches $68 \mathrm{meV} \approx 700 k_{B} T_{c}$. This result was indirectly verified by a photoemission study, which found that the energy spectrum is shifted noticeably towards higher binding energies at low temperatures as compared to room temperature ${ }^{24}$ Therefore, $\mathrm{Cd}_{2} \mathrm{Re}_{2} \mathrm{O}_{7}$ should be considered in the same category as $\mathrm{CePt}_{3} \mathrm{Si}$ and UIr. Moreover, $\mathrm{Cd}_{2} \mathrm{Re}_{2} \mathrm{O}_{7}$ is a useful test system for the theory since its superconducting properties are well established. ${ }^{25,26}$ The theory must, therefore, include the possibility for a nodeless $s$-wave-like order parameter ${ }^{27}$ Another appealing feature of $\mathrm{Cd}_{2} \mathrm{Re}_{2} \mathrm{O}_{7}$ is the relative simplicity of its normal metallic state: it neither orders magnetically, ${ }_{2}^{26}$ nor demonstrates Kondo-like behaviour as that found in $\mathrm{CePt}_{3} \mathrm{Si}^{8}$

In this Article, we consider general symmetry properties of the superconducting state in noncentrosymmetric crystals in which the degeneracy is lifted by strong SOC. As a first approximation, we restrict consideration to a one-band model, neglecting possible pairing in other bands and inter-band interactions. Sec. II discusses the symmetry properties of the superconducting order parameter. In Sec. III we consider Josephson tunneling between a conventional superconductor and a superconductor with nondegenerate bands. Sec. IV discusses the limiting case of small SOC, using the Rashba Hamiltonian as a specific example. In Sec. [ w we discuss the results of our approach in relation to previous theoretical developments and the applicability to real materials. 


\section{SUPERCONDUCTING ORDER PARAMETER}

Throughout this paper we use the weak coupling approach to treat the pairing interaction. While this approach offers no insight about the pairing mechanism, it has the advantage that many results can be obtained exactly. Also, the symmetry properties of the SC state remain essentially the same as in strong coupling models $?$

\section{A. Single-particle Hamiltonian}

In the condensed state it is normally sufficient to treat relativistic effects by the Pauli approximation to Dirac's fully relativistic approach. We begin by briefly reviewing the properties of the single-particle Hamiltonian ${ }^{4}$

$$
H_{1}=\frac{\mathbf{p}^{2}}{2 m}+V(\mathbf{r})+\frac{1}{4 m^{2} c^{2}}(\nabla V(\mathbf{r}) \times \mathbf{p} \cdot \boldsymbol{\sigma}),
$$

where $\mathbf{p}=-i \nabla$ is the momentum operator in the coordinate representation, $V(\mathbf{r})$ is the periodic potential of the crystal lattice, and $\boldsymbol{\sigma}=\left(\sigma_{x}, \sigma_{y}, \sigma_{z}\right)$ are the Pauli matrices. Here and in the following we use the unit system in which $\hbar=k_{B}=1$. The space group of the crystal is defined as the set of operations $g$ (rotations, reflections and translations) acting on the real space coordinates $\mathbf{r}$ which leave $V(\mathbf{r})$ invariant,

$$
g: V(\mathbf{r})=V(g \mathbf{r})=V(\mathbf{r}) .
$$

Because of the last term in (1), the transformation properties of $H_{1}$ are expressed as

$$
g: H_{1}(\mathbf{r})=\mathcal{D}(g) H_{1}(g \mathbf{r}) \mathcal{D}(g)^{-1}=H_{1}(\mathbf{r})
$$

where $\mathcal{D}(g)$ is a $2 \times 2$ spin- $1 / 2$ rotation matrix. $\mathcal{D}(g)$ is defined as follows. If $g$ is a bare rotation about the axis $\mathbf{n}$ by an angle $\phi$, then

$$
\mathcal{D}(g)=\cos \frac{\phi}{2}-i \mathbf{n} \cdot \boldsymbol{\sigma} \sin \frac{\phi}{2} .
$$

If $g$ includes inversion $I, g=I g^{\prime}$, or a translation $\tau(\mathbf{R})$, $g=\tau(\mathbf{R}) g^{\prime}$, where $g^{\prime}$ is a bare rotation, then $\mathcal{D}(g)=$ $\mathcal{D}\left(g^{\prime}\right)$, since inversion and translations do not change the spinor components.

The solutions $\Psi(\mathbf{r})$ of the Shrödinger equation

$$
H_{1} \Psi(\mathbf{r})=\varepsilon \Psi(\mathbf{r})
$$

are two-component spinors which transform according to

$$
g: \Psi(\mathbf{r})=\mathcal{D}(g) \Psi(g \mathbf{r}) .
$$

In nonmagnetic crystals $V(\mathbf{r})$ does not depend on spin, and the Hamiltonian (10) is also invariant under time reversal $\mathcal{K}$,

$$
\mathcal{K}: H_{1}(\mathbf{r})=\left(-i \sigma_{y}\right) H_{1}^{*}(\mathbf{r})\left(-i \sigma_{y}\right)^{-1}=H_{1}(\mathbf{r}),
$$

which uses the fact that spin reverses sign under time reversal, expressed by $\sigma_{y} \boldsymbol{\sigma}^{*} \sigma_{y}=-\boldsymbol{\sigma}$. Correspondingly,

$$
\mathcal{K}: \Psi(\mathbf{r})=\left(-i \sigma_{y}\right) \Psi^{*}(\mathbf{r}) .
$$

Further, if $\tau(\mathbf{R})$ is a proper lattice translation,

$$
\tau(\mathbf{R}): H_{1}(\mathbf{r})=H_{1}(\mathbf{r}+\mathbf{R})=H_{1}(\mathbf{r}) .
$$

Therefore, as well as in the case of vanishing SOC, the solutions of the Shrödinger equation (5) are Bloch waves labeled by a quantum number quasimomentum $\mathbf{k}$,

$$
\Psi_{\mathbf{k}}(\mathbf{r})=U_{\mathbf{k}}(\mathbf{r}) e^{i \mathbf{k r}},
$$

where $U_{\mathbf{k}}(\mathbf{r})$ is the periodic spinor

$$
U_{\mathbf{k}}(\mathbf{r})=\left(\begin{array}{c}
u_{\mathbf{k}, \uparrow}(\mathbf{r}) \\
u_{\mathbf{k}, \downarrow}(\mathbf{r})
\end{array}\right), \quad U_{\mathbf{k}}(\mathbf{r}+\mathbf{R})=U_{\mathbf{k}}(\mathbf{r}) .
$$

It follows from Eq. (6) that

$$
\begin{aligned}
g: \Psi_{\mathbf{k}}(\mathbf{r}) & =\mathcal{D}(g) U_{\mathbf{k}}(g \mathbf{r}) e^{i(\mathbf{k}, g \mathbf{r})} \\
& =\mathcal{D}(g) U_{\mathbf{k}}(g \mathbf{r}) e^{i\left(g^{-1} \mathbf{k}, \mathbf{r}\right)} \equiv \Psi_{g^{-1} \mathbf{k}}^{(g)}(\mathbf{r})
\end{aligned}
$$

Clearly, $\Psi_{g^{-1} \mathbf{k}}^{(g)}(\mathbf{r})$ is a solution of (5) $)$, corresponding to the quasimomentum $g^{-1} \mathbf{k}$. On the other hand, the same can be said about the function $\Psi_{g^{-1} \mathbf{k}}(\mathbf{r})$ obtained from $\Psi_{\mathbf{k}}(\mathbf{r})$ by the bare replacement $\mathbf{k} \rightarrow g^{-1} \mathbf{k}$. If the spin degeneracy of the bands is lifted, these two functions can only differ by a phase factor that does not depend on $\mathbf{r}$,

$$
\Psi_{g^{-1} \mathbf{k}}^{(g)}(\mathbf{r})=\exp \left[i \alpha_{g}(\mathbf{k})\right] \Psi_{g^{-1} \mathbf{k}}(\mathbf{r}) .
$$

This argument also applies to time reversal operation,

$$
\mathcal{K}: \Psi_{\mathbf{k}}(\mathbf{r})=\left(-i \sigma_{y}\right) U_{\mathbf{k}}^{*}(\mathbf{r}) e^{-i \mathbf{k r}} \equiv \Psi_{-\mathbf{k}}^{(\mathcal{K})}(\mathbf{r}) .
$$

Let us define $t(\mathbf{k})$ as the phase factor in

$$
\Psi_{-\mathbf{k}}^{(\mathcal{K})}(\mathbf{r})=t(\mathbf{k}) \Psi_{-\mathbf{k}}(\mathbf{r}), \quad t^{*}(\mathbf{k})=t^{-1}(\mathbf{k}) .
$$

Using the antilinear property of the time reversal operator $\mathcal{K}: a \Psi_{\mathbf{k}}(\mathbf{r})=a^{*} \mathcal{K}: \Psi_{\mathbf{k}}(\mathbf{r})$ and the fermionic nature of $\Psi_{\mathbf{k}}(\mathbf{r})$, one obtains,

$$
\begin{aligned}
\mathcal{K}^{2}: \Psi_{\mathbf{k}}(\mathbf{r}) & =-\Psi_{\mathbf{k}}(\mathbf{r}) \\
& =t^{*}(\mathbf{k}) \mathcal{K}: \Psi_{-\mathbf{k}}(\mathbf{r}) \\
& =t^{*}(\mathbf{k}) t(-\mathbf{k}) \Psi_{\mathbf{k}}(\mathbf{r})
\end{aligned}
$$

Thus, $t(\mathbf{k})$ is an odd function of $\mathbf{k}$,

$$
t(-\mathbf{k})=-t(\mathbf{k})
$$

Before discussing the interaction part of the Hamiltonian, we formulate the symmetry properties of the Hamiltonian (1) using the language of second quantization [See, e.g., Refs. 2829$]$. The $N$-particle wave function $\Phi_{N}$ is the anti-symmetrized product of $N$ single-particle functions $\Psi_{\mathbf{k}}(\mathbf{r})$ taken at $N$ different points $\mathbf{k}$. By writing 
$\left(\ldots, 1_{\mathbf{k}}, \ldots\right)$ as the argument of $\Phi_{N}$ we emphasize that it describes a state in which a single-particle state with quantum number $\mathbf{k}$ is filled. By definition, the creation operator $c_{\mathbf{k}}^{\dagger}$,

$$
c_{\mathbf{k}}^{\dagger} \Phi_{N-1}\left(\ldots, 0_{\mathbf{k}}, \ldots\right)= \pm \Phi_{N}\left(\ldots, 1_{\mathbf{k}}, \ldots\right),
$$

where the sign is defined by the antisymmetrization. In particular, we note that the matrix elements of the creation operator are real numbers. Using the definition (18) and the transformation properties of $\Psi_{\mathbf{k}}(\mathbf{r})$, we obtain ${ }^{30}$

$$
\begin{aligned}
g: c_{\mathbf{k}}^{\dagger} & =\exp \left[i \alpha_{g}(\mathbf{k})\right] c_{g^{-1}}^{\dagger}, \\
g: c_{\mathbf{k}} & =\exp \left[-i \alpha_{g}(\mathbf{k})\right] c_{g^{-1} \mathbf{k}} \\
\mathcal{K}: c_{\mathbf{k}}^{\dagger} & =t(\mathbf{k}) c_{-\mathbf{k}}^{\dagger} \equiv c_{-\mathbf{k}}^{\dagger(\mathcal{K})} \\
\mathcal{K}: c_{\mathbf{k}} & =t^{*}(\mathbf{k}) c_{-\mathbf{k}} \\
U_{1}(\theta): c_{\mathbf{k}}^{\dagger} & =e^{-i \theta} c_{\mathbf{k}}^{\dagger} \\
U_{1}(\theta): c_{\mathbf{k}} & =e^{i \theta} c_{\mathbf{k}}
\end{aligned}
$$

where $U_{1}(\theta)$ is a gauge transformation. The transformation properties of $c_{\mathbf{k}}$ follow from the fact that the number operator $c_{\mathbf{k}}^{\dagger} c_{\mathbf{k}}$ must be a scalar.

The one-band single-particle Hamiltonian can be expressed as

$$
\mathcal{H}_{1}=\sum_{\mathbf{k}} \xi_{\mathbf{k}} c_{\mathbf{k}}^{\dagger} c_{\mathbf{k}}
$$

where $\xi_{\mathbf{k}}=\varepsilon_{\mathbf{k}}-\mu, \varepsilon_{\mathbf{k}}$ is the eigenvalue of the Hamiltonian (11) corresponding to momentum $\mathbf{k}$, and $\mu$ is the chemical potential. We note that $c_{\mathbf{k}}^{\dagger}$ and $c_{\mathbf{k}}$ are both two component spinors, and have all the general properties of spin- $\frac{1}{2}$ fermion operators, even though additional quantum numbers (such as spin) are omitted since we consider only one band.

\section{B. Pairing term}

If the interband interaction is neglected, the interaction term is

$$
\mathcal{H}_{\text {pair }}=\frac{1}{2} \sum_{\mathbf{k}_{1}, \mathbf{k}_{2}} V_{2}\left(\mathbf{k}_{1}, \mathbf{k}_{2}\right) c_{-\mathbf{k}_{1}}^{\dagger} c_{\mathbf{k}_{1}}^{\dagger} c_{\mathbf{k}_{2}} c_{-\mathbf{k}_{2}},
$$

where $V_{2}\left(\mathbf{k}_{1}, \mathbf{k}_{2}\right)=\left\langle\Psi_{-\mathbf{k}_{1}} \Psi_{\mathbf{k}_{1}}\left|\widehat{V}_{2}\right| \Psi_{-\mathbf{k}_{2}} \Psi_{\mathbf{k}_{2}}\right\rangle$ is the twoparticle matrix element. In the weak-coupling approach, 7 one introduces the mean field potential

$$
\Delta(\mathbf{k})=-\sum_{\mathbf{k}^{\prime}} V_{2}\left(\mathbf{k}, \mathbf{k}^{\prime}\right)\left\langle c_{\mathbf{k}^{\prime}} c_{-\mathbf{k}^{\prime}}\right\rangle
$$

and the interaction (21) is approximated by

$$
\mathcal{H}_{2}=\frac{1}{2} \sum_{\mathbf{k}}\left[\Delta(\mathbf{k}) c_{\mathbf{k}}^{\dagger} c_{-\mathbf{k}}^{\dagger}+\Delta^{*}(\mathbf{k}) c_{-\mathbf{k}} c_{\mathbf{k}}\right]
$$

where a constant term is neglected. It follows immediately from the anticommutation of the fermion operators that

$$
\Delta(-\mathbf{k})=-\Delta(\mathbf{k})
$$

One can derive the other transformation properties of $\Delta(\mathbf{k})$ from the fact that the full Hamiltonian $\mathcal{H}=$ $\mathcal{H}_{1}+\mathcal{H}_{2}$ is invariant under space group operations, timereversal and gauge transformations. Using (19), one obtains

$$
g: \Delta(\mathbf{k})=\Delta\left(g^{-1} \mathbf{k}\right) \exp \left[-i \alpha_{g}(\mathbf{k})-i \alpha_{g}(-\mathbf{k})\right]
$$

It will be seen already from a simple example given in Sec. IV that the phase factor in Eq. (25) is not trivial. In general, its dependence on $g$ and $\mathbf{k}$ cannot be eliminated. Therefore, the function $\Delta(\mathbf{k})$ does not transform according to irreducible representations of the space (point) group. For crystals with a centre of symmetry, a comprehensive discussion of this property has been given by Blount ${ }^{31}$ Instead of the pairing potential $\Delta_{\mu \nu}$ which transforms like $\left\langle\psi_{\mu}, \psi_{\nu}\right\rangle$, an auxiliary object which transforms like $\left\langle\mathcal{K}: \psi_{\mu}, \psi_{\nu}\right\rangle$ is introduced. Here $\mu$ and $\nu$ denote the set of single-particle quantum numbers. Following this idea, we define function $\chi(\mathbf{k})$ by

$$
\Delta(\mathbf{k})=\chi(\mathbf{k}) t(\mathbf{k})
$$

Then, using the definition of $c_{-\mathbf{k}}^{\dagger(\mathcal{K})}$ from the third Eq. (19) the first term in $\mathcal{H}_{2}$ is expressed as

$$
\frac{1}{2} \sum_{\mathbf{k}} \chi(\mathbf{k}) t(\mathbf{k}) c_{\mathbf{k}}^{\dagger} c_{-\mathbf{k}}^{\dagger}=\frac{1}{2} \sum_{\mathbf{k}} \chi(\mathbf{k}) c_{\mathbf{k}}^{\dagger} c_{-\mathbf{k}}^{\dagger(\mathcal{K})}
$$

Using the commutation property $g \mathcal{K}=\mathcal{K} g$, it is straightforward to show that ${ }^{10} g: c_{-\mathbf{k}}^{\dagger(\mathcal{K})}=\exp \left[-i \alpha_{g}(\mathbf{k})\right] c_{-g^{-1} \mathbf{k}}^{\dagger(\mathcal{K})}$. Thus, from the invariance of (27), we find

$$
g: \chi(\mathbf{k})=\chi\left(g^{-1} \mathbf{k}\right) .
$$

Eq. (28) is the basis of the group-theoretical classification of SC states in noncentrosymmetric crystals. $\chi(\mathbf{k})$ can be expanded in terms of basis functions $\chi_{i}(\mathbf{k})$ of irreducible representations of the space (point) group. At this point we restrict our consideration to homogeneous $\mathrm{SC}$ states for simplicity, so that only the point group is involved,

$$
\chi(\mathbf{k})=\sum_{i} \eta_{i} \chi_{i}(\mathbf{k})
$$

where $\eta_{i}$ will be identified as the components of the order parameter. It follows from Eqs. (17), (24) and (26) that $\chi(-\mathbf{k})=\chi(\mathbf{k})$. Examples of even basis functions $\chi_{i}(\mathbf{k})$ for the irreducible representations of point groups $C_{4 v}$ for $\mathrm{CePt}_{3} \mathrm{Si}$ and $C_{2}$ for UIr are given in Table 1

The other transformation properties of $\chi(\mathbf{k})$ also follow from (19) and (27),

$$
\mathcal{K}: \chi(\mathbf{k})=\chi^{*}(\mathbf{k}), \quad U_{1}(\theta): \chi(\mathbf{k})=e^{2 i \theta} \chi(\mathbf{k})
$$


TABLE I: Even basis functions for irreducible representations (IR) of point groups $C_{4 v}$ and $C_{2}$. The notations for IR and their character tables can be found in Ref. 28. $a, b$, and $c$ denote lattice constants.

\begin{tabular}{lll}
\hline \hline IR & Non-periodic & Periodic \\
\hline$C_{4 v}$ & & \\
$A_{1}$ & $1, k_{x}^{2}+k_{y}^{2}, k_{z}^{2}$ & $\cos k_{x} a+\cos k_{y} a, \cos k_{z} c$ \\
$A_{2}$ & $k_{x} k_{y}\left(k_{x}^{2}-k_{y}^{2}\right)$ & $\sin k_{x} a \sin k_{y} a\left(\cos k_{x} a-\cos k_{y} a\right)$ \\
$B_{1}$ & $k_{x}^{2}-k_{y}^{2}$ & $\cos k_{x} a-\cos k_{y} a$ \\
$B_{2}$ & $k_{x} k_{y}$ & $\sin k_{x} a \sin k_{y} a$ \\
$E$ & $\left\{\begin{array}{l}k_{x} k_{z} \\
k_{y} k_{z}\end{array}\right.$ & $\left\{\begin{array}{l}\sin k_{x} a \sin k_{z} c \\
\sin k_{y} a \sin k_{z} c\end{array}\right.$ \\
\hline$C_{2}$ & & $\cos k_{x} a, \cos k_{y} b, \cos k_{z} c$ \\
$A_{1}$ & $1, k_{x}^{2}, k_{y}^{2}, k_{z}^{2}$, & $\sin k_{x} a \sin k_{y} b$ \\
& $k_{x} k_{y}$ & $\sin k_{x} a \sin k_{z} c, \sin k_{y} b \sin k_{z} c$ \\
$A_{2}$ & $k_{x} k_{z}, k_{y} k_{z}$ & \\
\hline \hline
\end{tabular}

where we again use antilinearity of $\mathcal{K}$. As is usually done in the Ginzburg-Landau approach, the transformation properties of $\chi(\mathbf{k})$ are reformulated as transformations of $\eta_{i}, \underline{7}$ If $\chi_{i}(\mathbf{k})$ are chosen real, then $g: \eta_{i}=D_{i k} \eta_{k}$, $\mathcal{K}: \eta_{i}=\eta_{i}^{*}$ and $U_{1}(\theta): \eta_{i}=e^{2 i \theta} \eta_{i}$, where $D$ is the matrix corresponding to $g$ in the representation chosen.

\section{Possible forms of the Ginzburg-Landau potential for $\mathrm{CePt}_{3} \mathrm{Si}$}

Even though $C_{4 v}$ lacks inversion symmetry, the homogeneous terms in the Ginzburg-Landau potential (GLP) coincide with those of $D_{4 h}, \frac{7}{4}$ because the GLP has to be invariant with respect to gauge transformations, in particular $U_{1}(\pi / 2): \chi(\mathbf{k})=-\chi(\mathbf{k})$. For every representation $\Gamma$ listed in Table \ the product $\Gamma \otimes \Gamma^{*}$ contains the representation $A_{1}$. The $z$-component of the gradient operator

$$
\mathbf{D}=\nabla-\frac{2 i e}{c} \mathbf{A}
$$

also transforms according to $A_{1}$, therefore the GLP density contains a term linear in $D_{z}$,

$$
\sum_{i} \eta_{i}^{*} D_{z} \eta_{i}+\eta_{i} D_{z}^{*} \eta_{i}^{*}
$$

However, this terms is equal to the derivative $\frac{\partial}{\partial z} \sum_{i}\left|\eta_{i}\right|^{2}$. Hence, after integration over the sample, its contribution to the GLP vanishes.

For all one-dimensional representations of $C_{4 v}$, the GLP is

$$
\begin{aligned}
F_{1 D}=F_{0}+\int & d \mathbf{r}\left[K_{1}\left(\left|D_{x} \eta\right|^{2}+\left|D_{y} \eta\right|^{2}\right)+K_{2}\left|D_{z} \eta\right|^{2}\right. \\
& \left.+\alpha|\eta|^{2}+\beta|\eta|^{4}\right]
\end{aligned}
$$

For the two-dimensional representation $E$,

$$
\begin{aligned}
& F_{2 D}=F_{0}+\int d \mathbf{r}\left\{K_{1}\left(\left|D_{x} \eta_{1}\right|^{2}+\left|D_{y} \eta_{2}\right|^{2}\right)\right. \\
& +K_{2}\left(\left|D_{x} \eta_{2}\right|^{2}+\left|D_{y} \eta_{1}\right|^{2}\right)+K_{3}\left[\left(D_{x} \eta_{1}\right)\left(D_{y} \eta_{2}\right)^{*}+\text { c.c. }\right] \\
& +K_{4}\left[\left(D_{x} \eta_{2}\right)\left(D_{y} \eta_{1}\right)^{*}+\text { c.c. }\right]+K_{5}\left(\left|D_{z} \eta_{1}\right|^{2}+\left|D_{z} \eta_{2}\right|^{2}\right) \\
& +\alpha\left(\left|\eta_{1}\right|^{2}+\left|\eta_{2}\right|^{2}\right)+\beta_{1}\left(\left|\eta_{1}\right|^{2}+\left|\eta_{2}\right|^{2}\right)^{2}+\beta_{2}\left(\eta_{1}^{*} \eta_{2}-\eta_{1} \eta_{2}^{*}\right)^{2} \\
& \left.+\beta_{3}\left|\eta_{1}\right|^{2}\left|\eta_{2}\right|^{2}\right\} .
\end{aligned}
$$

\section{Green's functions}

The most straightforward way to calculate the normal $G(\mathbf{k}, \tau)$ and anomalous $F(\mathbf{k}, \tau), F^{\dagger}(\mathbf{k}, \tau)$ Green's functions within the weak-coupling approach is to use the Gor'kov equations. ${ }^{32,33}$ The Green's functions are defined as follows,

$$
\begin{aligned}
G(\mathbf{k}, \tau) & =-\left\langle T_{\tau}\left\{c_{\mathbf{k}}(\tau) c_{\mathbf{k}}^{\dagger}(0)\right\}\right\rangle \\
F(\mathbf{k}, \tau) & =\left\langle T_{\tau}\left\{c_{\mathbf{k}}(\tau) c_{-\mathbf{k}}(0)\right\}\right\rangle \\
F^{\dagger}(\mathbf{k}, \tau) & =\left\langle T_{\tau}\left\{c_{-\mathbf{k}}^{\dagger}(\tau) c_{\mathbf{k}}^{\dagger}(0)\right\}\right\rangle
\end{aligned}
$$

where $T_{\tau}$ is the imaginary time ordering operator. The Gor'kov equations for the Fourier transforms are

$$
\begin{aligned}
{\left[i \omega_{n}-\xi_{\mathbf{k}}\right] G\left(\mathbf{k}, \omega_{n}\right)+\Delta(\mathbf{k}) F^{\dagger}\left(\mathbf{k}, \omega_{n}\right) } & =1 \\
{\left[i \omega_{n}+\xi_{\mathbf{k}}\right] F^{\dagger}\left(\mathbf{k}, \omega_{n}\right)+\Delta^{*}(\mathbf{k}) G\left(\mathbf{k}, \omega_{n}\right) } & =0
\end{aligned}
$$

where $\omega_{n}=\pi T(2 n+1)$ are the Matsubara frequencies for fermions. The equations are easily solved by

$$
\begin{aligned}
G\left(\mathbf{k}, \omega_{n}\right) & =-\left(i \omega_{n}+\xi_{\mathbf{k}}\right) /\left(\omega_{n}^{2}+\xi_{\mathbf{k}}^{2}+|\chi(\mathbf{k})|^{2}\right), \\
F^{\dagger}\left(\mathbf{k}, \omega_{n}\right) & =\chi^{*}(\mathbf{k}) t^{*}(\mathbf{k}) /\left(\omega_{n}^{2}+\xi_{\mathbf{k}}^{2}+|\chi(\mathbf{k})|^{2}\right),
\end{aligned}
$$

where we have made use of the fact that $|\Delta(\mathbf{k})|^{2}=$ $|\chi(\mathbf{k})|^{2}$.

Gor'kov and Rashba ${ }^{34}$ considered a model of superconductivity with split bands due to SOC and an isotropic pairing interaction. They showed that the thermodynamics of a such a superconductor is equivalent to that of a conventional $s$-wave superconductor. Here we have generalized this result to the anisotropic case. As is evident from the denominator in Eq. (37), the thermodynamics of a noncentrosymmetric superconductor are governed by the gap $|\chi(\mathbf{k})|$.

\section{JOSEPHSON EFFECT}

We consider tunneling of SC electrons between a superconductor with non-degenerate bands and a light conventional superconducting metal like $\mathrm{Nb}$, in which electronic bands are spin degenerate. The tunneling Hamiltonian is

$$
\mathcal{H}_{T}=\sum_{\mathbf{k}_{1}, \mathbf{k}_{2}, s}\left[T_{s}\left(\mathbf{k}_{1}, \mathbf{k}_{2}\right) c_{\mathbf{k}_{1}}^{\dagger} a_{\mathbf{k}_{2}, s}+T_{s}^{*}\left(\mathbf{k}_{1}, \mathbf{k}_{2}\right) a_{\mathbf{k}_{2}, s}^{\dagger} c_{\mathbf{k}_{1}}\right],
$$


where, $a_{\mathbf{k}, s}^{\dagger}$ creates an electron with spin $s$ in the conventional superconductor and $T_{s}\left(\mathbf{k}_{1}, \mathbf{k}_{2}\right)$ is the tunneling matrix element. By applying the time reversal operation to the first term in $\mathcal{H}_{T}$, we obtain

$$
\begin{aligned}
& \sum_{\mathbf{k}_{1}, \mathbf{k}_{2}, s, s^{\prime}} T_{s}^{*}\left(\mathbf{k}_{1}, \mathbf{k}_{2}\right) t\left(\mathbf{k}_{1}\right) c_{-\mathbf{k}_{1}}^{\dagger}\left(-i \sigma_{y}\right)_{s s^{\prime}} a_{-\mathbf{k}_{2}, s^{\prime}} \\
= & \sum_{\mathbf{k}_{1}, \mathbf{k}_{2}, s, s^{\prime}} t\left(-\mathbf{k}_{1}\right)\left(i \sigma_{y}\right)_{s s^{\prime}} T_{s^{\prime}}^{*}\left(-\mathbf{k}_{1},-\mathbf{k}_{2}\right) c_{\mathbf{k}_{1}}^{\dagger} a_{\mathbf{k}_{2}, s}
\end{aligned}
$$

Therefore,

$$
T_{s}\left(-\mathbf{k}_{1},-\mathbf{k}_{2}\right)=t\left(\mathbf{k}_{1}\right) \sum_{s^{\prime}}\left(i \sigma_{y}\right)_{s s^{\prime}} T_{s^{\prime}}^{*}\left(\mathbf{k}_{1}, \mathbf{k}_{2}\right)
$$

The following derivation follows that for conventional superconductors given in Refs. 7, 33, which is in turn based on the formalism originally proposed by Ambegaokar and Baratoff 35 For simplicity, we restrict our consideration to the case of zero voltage. The current flowing between the two superconductors is $j=e\langle\partial N(t) / \partial t\rangle=$ ei $\left\langle\left[\mathcal{H}_{T}, N\right]\right\rangle$, where $N(t)=e^{i \mathcal{H}_{T} t} \sum_{\mathbf{k}} c_{\mathbf{k}}^{\dagger} c_{\mathbf{k}} e^{-i \mathcal{H}_{T} t}$ is the time-dependent particle number operator.

Following the same steps as described in Ref. 33, we obtain

$$
j=2 e T \operatorname{Im} \sum_{\mathbf{k}_{1}, \mathbf{k}_{2}, s, s^{\prime}, n} T_{s}\left(\mathbf{k}_{1}, \mathbf{k}_{2}\right) T_{s^{\prime}}\left(-\mathbf{k}_{1},-\mathbf{k}_{2}\right) F^{\dagger}\left(\mathbf{k}_{1}, \omega_{n}\right) F_{s s^{\prime}}^{(c)}\left(\mathbf{k}_{2}, \omega_{n}\right),
$$

where $F_{s s^{\prime}}^{(c)}\left(\mathbf{k}, \omega_{n}\right)=\psi(\mathbf{k})(i \sigma)_{s s^{\prime}} /\left(\omega_{n}^{2}+\epsilon_{\mathbf{k}}^{2}+|\psi(\mathbf{k})|^{2}\right)$ is the anomalous Green's function for a conventional superconductor with the single-particle spectrum $\epsilon_{\mathbf{k}}$ and singlet gap function $\psi(\mathbf{k}) ! \frac{7}{2}$ Using (37) and (40), we obtain

$$
j=-2 e T \operatorname{Im} \sum_{\mathbf{k}_{1}, \mathbf{k}_{2}} \sum_{n} \frac{\chi^{*}\left(\mathbf{k}_{1}\right) \psi\left(\mathbf{k}_{2}\right)}{\left(\omega_{n}^{2}+\xi_{\mathbf{k}_{1}}^{2}+\left|\chi\left(\mathbf{k}_{1}\right)\right|^{2}\right)\left(\omega_{n}^{2}+\epsilon_{\mathbf{k}_{2}}^{2}+\left|\psi\left(\mathbf{k}_{2}\right)\right|^{2}\right)} \sum_{s}\left|T_{s}\left(\mathbf{k}_{1}, \mathbf{k}_{2}\right)\right|^{2} .
$$

The sum over Matsubara frequencies can be easily calculated if needed by using the formula $2 T \sum_{n}\left(\omega_{n}^{2}+E^{2}\right)^{-1}=$ $\tanh (E / 2 T) / E$. Therefore, the final expression for the Josephson current does not depend on $t(\mathbf{k})$. Once again, we see that the superconductor with SOC split bands behaves as a singlet superconductor with gap function $\chi(\mathbf{k})$.

The tunneling Hamiltonian $\mathcal{H}_{T}$ is invariant with respect to the operations $g$ of the point group which leave the surface invariant. Thus,

$$
g: T_{s}\left(\mathbf{k}_{1}, \mathbf{k}_{2}\right)=e^{-i \alpha_{g}\left(\mathbf{k}_{1}\right)} \mathcal{D}(g)_{s s^{\prime}} T_{s^{\prime}}\left(g^{-1} \mathbf{k}_{1}, g^{-1} \mathbf{k}_{2}\right)
$$

It follows that

$$
g: \sum_{s}\left|T_{s}\left(\mathbf{k}_{1}, \mathbf{k}_{2}\right)\right|^{2}=\sum_{s}\left|T_{s}\left(g^{-1} \mathbf{k}_{1}, g^{-1} \mathbf{k}_{2}\right)\right|^{2}
$$

Therefore, $j$ is invariant under the operations $g$ as expected.

\section{LIMIT OF SMALL SPIN-ORBIT COUPLING}

In order to verify the general results given in Sec. II, it is important to show that they remain valid for crystals with small SOC, i.e. for almost degenerate electronic bands. In this Section we consider the Rashba approximation, 36 because (i) the one particle Hamiltonian is then exactly solvable and (ii) we can easily compare our results for superconductivity with those known from the literature, where the Rashba approximation was used. 34,37

In the Rashba approximation, one assumes that the solutions of the Hamiltonian $H_{0}=\mathbf{p}^{2} / 2 m+V(\mathbf{r})$ are plain waves with definite projections of spin and energy spectrum $\varepsilon_{\mathbf{k}}^{0}$, and that $\nabla V(\mathbf{r})$ can be replaced by a constant vector directed along the axis of symmetry $\mathbf{n}=(001)$. Therefore Eq. (1) is approximated by

$$
H_{1}^{\prime}=\left(\begin{array}{cc}
\varepsilon_{\mathbf{k}}^{0} & -\beta\left(k_{y}+i k_{x}\right) \\
-\beta\left(k_{y}-i k_{x}\right) & \varepsilon_{\mathbf{k}}^{0}
\end{array}\right)
$$

where $\beta$ is a constant which characterizes the strength of the SOC. The Hamiltonian is diagonalized by the spinors 34

$$
\Psi_{\mathbf{k}, \pm}=\frac{1}{\sqrt{2 V}}\left(\begin{array}{c}
1 \\
\pm i \exp \left(i \varphi_{\mathbf{k}}\right)
\end{array}\right) e^{i \mathbf{k r}}
$$

corresponding to the eigenvalues $\varepsilon_{\mathbf{k}}^{ \pm}=\varepsilon_{\mathbf{k}}^{0} \pm \beta\left|\mathbf{k}_{\perp}\right|$. Here, $\exp \left(i \varphi_{\mathbf{k}}\right)=\left(k_{x}+i k_{y}\right) /\left|\mathbf{k}_{\perp}\right|$ and $\left|\mathbf{k}_{\perp}\right|=\sqrt{k_{x}^{2}+k_{y}^{2}}$. In the following, we shall consider coupling in ' + ' band as an example.

In order to illustrate the complicated transformation properties (25) of $\Delta(\mathbf{k})$, let us consider as an example $g=m_{x}$, the mirror plane perpendicular to $x$-axis. Acting 
by $m_{x}$ on $\Psi_{\mathbf{k}+}$, we obtain

$$
\begin{aligned}
& \frac{1}{\sqrt{2 V}}\left(\begin{array}{cc}
0 & -i \\
-i & 0
\end{array}\right)\left(\begin{array}{c}
1 \\
i e^{i \varphi_{\mathbf{k}}}
\end{array}\right) e^{i\left(\mathbf{k}, m_{x} \mathbf{r}\right)} \\
& \quad=\frac{e^{i \varphi_{\mathbf{k}}}}{\sqrt{2 V}}\left(\begin{array}{c}
1 \\
i e^{i \varphi_{m_{x} \mathbf{k}}}
\end{array}\right) e^{i\left(m_{x} \mathbf{k}, \mathbf{r}\right)}=e^{i \varphi_{\mathbf{k}}} \Psi_{m_{x} \mathbf{k},+}
\end{aligned}
$$

where $\exp \left(i \varphi_{m_{x} \mathbf{k}}\right)=-\exp \left(-i \varphi_{\mathbf{k}}\right)$ was used. Hence, the phase factor defined by Eq. (25) is equal to $\left(i k_{x}+\right.$ $\left.k_{y}\right)^{2} / \mathbf{k}_{\perp}^{2}$. find

By applying time reversal to $\Psi_{\mathbf{k},+}$ using Eq. (14), we

$$
t(\mathbf{k})=i \exp \left(-i \varphi_{\mathbf{k}}\right)
$$

The fermion operators corresponding to initial spin-up and spin-down states can be expressed in terms of the new band operators as

$$
c_{\mathbf{k} \uparrow}^{\dagger}=\frac{1}{\sqrt{2}}\left(c_{\mathbf{k}+}^{\dagger}+c_{\mathbf{k}-}^{\dagger}\right), \quad c_{\mathbf{k} \downarrow}^{\dagger}=-\frac{i e^{-i \varphi_{\mathbf{k}}}}{\sqrt{2}}\left(c_{\mathbf{k}+}^{\dagger}-c_{\mathbf{k}-}^{\dagger}\right) .
$$

At this point we assume that before SOC has been turned on, the electrons were paired in the singlet state with gap function $\psi(\mathbf{k})$,

$$
\mathcal{H}_{2}^{\prime}=\frac{1}{2} \sum_{\mathbf{k}} \psi(\mathbf{k}) c_{\mathbf{k} \uparrow}^{\dagger} c_{-\mathbf{k} \downarrow}^{\dagger}+\text { h.c. }
$$

Using (49) and (48), we obtain

$$
\mathcal{H}_{2}^{\prime}=\frac{1}{4} \sum_{\mathbf{k}} t(\mathbf{k}) \psi(\mathbf{k}) c_{\mathbf{k}+}^{\dagger} c_{-\mathbf{k}+}^{\dagger}+\text { h.c. }+\ldots
$$

where the rest of the terms, which describe pairing in the other band and inter-band pairing, are neglected. By comparing (51) with (23) we obtain $\Delta(\mathbf{k})$ in the form (26) with $\chi(\mathbf{k})=\psi(\mathbf{k}) / 2$.

Interestingly, a similar result is obtained if one starts with triplet pairing for spin states, $\stackrel{7}{?}$

$$
\begin{aligned}
\mathcal{H}_{2}^{\prime} & =\frac{1}{2} \sum_{\mathbf{k}}\left\{\left[-d_{x}(\mathbf{k})+i d_{y}(\mathbf{k})\right] c_{\mathbf{k} \uparrow}^{\dagger} c_{-\mathbf{k} \uparrow}^{\dagger}+\left[d_{x}(\mathbf{k})+i d_{y}(\mathbf{k})\right] c_{\mathbf{k} \downarrow}^{\dagger} c_{-\mathbf{k} \downarrow}^{\dagger}+d_{z}(\mathbf{k})\left(c_{\mathbf{k} \uparrow}^{\dagger} c_{-\mathbf{k} \downarrow}^{\dagger}+c_{\mathbf{k} \downarrow}^{\dagger} c_{-\mathbf{k} \uparrow}^{\dagger}\right)\right\}+\text { h.c. } \\
& =\frac{1}{2} \sum_{\mathbf{k}} t(\mathbf{k})\left[d_{y}(\mathbf{k}) \hat{k}_{x}-d_{x}(\mathbf{k}) \hat{k}_{y}\right] c_{\mathbf{k}+}^{\dagger} c_{-\mathbf{k}+}^{\dagger}+\text { h.c. }+\ldots
\end{aligned}
$$

where $\hat{k}_{i}=k_{i} /\left|\mathbf{k}_{\perp}\right|$ and again, only terms corresponding coupling within the ' + ' band are kept. Therefore, we again find $\Delta(\mathbf{k})$ in the form (26) with

$$
\chi(\mathbf{k})=d_{y}(\mathbf{k}) \hat{k}_{x}-d_{x}(\mathbf{k}) \hat{k}_{y} .
$$

Frigeri et al ${ }^{37}$ used weak coupling theory to show that a phase transition to the triplet pairing state $\mathbf{d}(\mathbf{k})=$ $\left(-k_{y}, k_{x}, 0\right)$ (which transforms according to the representation $A_{2}$ ) in $\mathrm{CePt}_{3} \mathrm{Si}$ is not suppressed by small but finite $\beta$. According to (53), this corresponds to $\chi(\mathbf{k}) \propto \hat{k}_{x}^{2}+\hat{k}_{y}^{2}$, which is isotropic and fully gapped and transforms like $A_{1}$. By using the vectors $\mathbf{d}(\mathbf{k})$ corresponding to the rest of the irreducible representations of $C_{4 v}$ (which are similar to those for $D_{4 h}$ and are given in Ref. 7), the corresponding functions $\chi(\mathbf{k})$ may be obtained from Eq. (53) and are listed in Table Therefore, in general, starting from $\mathbf{d}(\mathbf{k})$ corresponding to a representation $\Gamma$, we generate $\chi(\mathbf{k})$ using Eq. (53) corresponding to another representation $\Gamma^{\prime}$, while in the singlet case, $\chi(\mathbf{k})$ and $\psi(\mathbf{k}) / 2$ belong to the same representation because they are proportional. The difference is due to the transformation properties of the paired state under spin rotation, which was used to diagonalize the Hamiltonian
$H_{1}^{\prime}$ : the singlet state transforms into itself as a scalar, whereas the triplet state transforms as a spin- 1 state ${ }^{38}$

TABLE II: Basis functions for triplet superconductivity in $C_{4 v}$ crystals with non-degenerate bands and vanishing SOC and the corresponding even basis functions determined by Eq. (53). Note that $\Delta(\mathbf{k})$ is defined by Eq. (26) even for the triplet case.

\begin{tabular}{llll}
\hline \hline$A_{1}$ & $\mathbf{d}(\mathbf{k})=\left(k_{x}, k_{y}, 0\right)$ & - & \\
& $\mathbf{d}(\mathbf{k})=\left(k_{x}^{3}, k_{y}^{3}, 0\right)$ & $\chi(\mathbf{k})=k_{x} k_{y}\left(k_{x}^{2}-k_{y}^{2}\right)$ & $A_{2}$ \\
$A_{2}$ & $\mathbf{d}(\mathbf{k})=\left(-k_{y}, k_{x}, 0\right)$ & $\chi(\mathbf{k})=k_{x}^{2}+k_{y}^{2}$ & $A_{1}$ \\
$B_{1}$ & $\mathbf{d}(\mathbf{k})=\left(k_{x},-k_{y}, 0\right)$ & $\chi(\mathbf{k})=k_{x} k_{y}$ & $B_{2}$ \\
$B_{2}$ & $\mathbf{d}(\mathbf{k})=\left(k_{y}, k_{x}, 0\right)$ & $\chi(\mathbf{k})=k_{x}^{2}-k_{y}^{2}$ & $B_{1}$ \\
$E$ & $\mathbf{d}_{1}(\mathbf{k})=\left(k_{z}, 0,0\right)$ & $\chi_{1}(\mathbf{k})=k_{y} k_{z}$ & $E$ \\
& $\mathbf{d}_{2}(\mathbf{k})=\left(0, k_{z}, 0\right)$ & $\chi_{2}(\mathbf{k})=k_{x} k_{z}$ & \\
\hline \hline
\end{tabular}




\section{DISCUSSION}

A close analogy between the superconductors under consideration and usual centrosymmetric singlet superconductors should not be surprising. A particle described by the wave function (10) which is a solution of Hamiltonian (1) has $\operatorname{spin} \mathbf{s}(\mathbf{k})=\frac{1}{2}\left\langle\Psi_{\mathbf{k}}|\boldsymbol{\sigma}| \Psi_{\mathbf{k}}\right\rangle$. Since $\Psi_{-\mathbf{k}}$ and $\Psi_{-\mathbf{k}}^{(\mathcal{K})}$ differ only by a constant phase factor, we have

$$
\begin{aligned}
\mathbf{s}(-\mathbf{k}) & =\frac{1}{2}\left\langle\Psi_{-\mathbf{k}}|\boldsymbol{\sigma}| \Psi_{-\mathbf{k}}\right\rangle=\frac{1}{2}\left\langle\Psi_{-\mathbf{k}}^{(\mathcal{K})}|\boldsymbol{\sigma}| \Psi_{-\mathbf{k}}^{(\mathcal{K})}\right\rangle \\
& =\mathcal{K}: \frac{1}{2}\left\langle\Psi_{\mathbf{k}}\left|\boldsymbol{\sigma}^{(\mathcal{K})}\right| \Psi_{\mathbf{k}}\right\rangle=\frac{1}{2}\left\langle\Psi_{\mathbf{k}}\left|\boldsymbol{\sigma}^{(\mathcal{K})}\right| \Psi_{\mathbf{k}}\right\rangle^{*} \\
& =-\frac{1}{2}\left\langle\Psi_{\mathbf{k}}|\boldsymbol{\sigma}| \Psi_{\mathbf{k}}\right\rangle=-\mathbf{s}(\mathbf{k}),
\end{aligned}
$$

where we have used the fact that diagonal matrix elements of the spin operator are real and $\boldsymbol{\sigma}^{(\mathcal{K})}=$ $\left(-i \sigma_{y}\right) \boldsymbol{\sigma}^{*}\left(-i \sigma_{y}\right)^{-1}=-\boldsymbol{\sigma}$. Therefore, particles with opposite momenta within the same band always have opposite spin [See also Ref. 11].

It was first noted by Anderson ${ }^{39}$ that for crystals in which either the momentum $\mathbf{k}$ or spin is not a good quantum number, as in dirty superconductors, a one particle state should be paired with its time reversal. Using this idea, Samokhin et al ${ }^{10}$ define $c_{-\mathbf{k}}^{\dagger}$ as time reversal of $c_{\mathbf{k}}^{\dagger}$. This in turn leads to the conclusion that $\Delta(\mathbf{k})$ is an odd function which transforms according to the irreducible representations of the point group. Then, the gaps in the quasiparticle spectrum of the superconducting states described by all one dimensional representations of $C_{4 v}$ have line nodes. This, in particular, rules out the possibility of an isotropic full gap, and therefore contradicts the theoretical results of Refs. 3437 and the experimental results for $\mathrm{Cd}_{2} \mathrm{Re}_{2} \mathrm{O}_{7}$. The origin of the discrepancy is that Anderson's statement is correct up to a phase factor $t(\mathbf{k})$, which is not important in the normal state due to gauge invariance but cannot be ignored in the superconducting state. ${ }^{40}$ In other words, one cannot define $c_{\mathbf{k}}^{\dagger}$ in an asymmetric unit of the Brillouin zone and then use the symmetry elements (including time reversal) to define the states in the rest of the Brillouin zone. Such a procedure would lead to a discontinuity of the phase of the wave function on the boundaries of the asymmetric units. Also, if $c_{\mathbf{k}}^{\dagger}$ is paired with $\mathcal{K} c_{\mathbf{k}}^{\dagger}$, then $\mathcal{K} c_{\mathbf{k}}^{\dagger}$ must be paired with $\mathcal{K}^{2} c_{\mathbf{k}}^{\dagger}=-c_{\mathbf{k}}^{\dagger}$, which is a contradiction. Instead, if $c_{\mathbf{k}}^{\dagger}$ describes the particle with momentum $\mathbf{k}$ and $\mathbf{k}^{\prime}=g^{-1} \mathbf{k}$, where $g$ is a point group element or time reversal, then the particle with momentum $\mathbf{k}^{\prime}$ must be described by $c_{\mathbf{k}^{\prime}}^{\dagger}=c_{g^{-1} \mathbf{k}}^{\dagger}$, which is proportional but not equal to $g: c_{\mathbf{k}}^{\dagger}$.

The formalism developed in this paper can be directly applied to $\mathrm{Cd}_{2} \mathrm{Re}_{2} \mathrm{O}_{7}$, which shows no sign of magnetic ordering and therefore possesses time reversal symmetry 26 The low temperature structure has symmetry $I 4_{1} 22, \frac{19}{1}$ with point group $D_{4}$, which is isomorphic to $C_{4 v}$. The superconducting order parameter corresponds to the representation $A_{1}$ since no nodes in the quasiparticle spectrum have been found.

In the application to $\mathrm{CePt}_{3} \mathrm{Si}$, this theory should be slightly modified to include the effect of antiferromagnetic ordering $\left(T_{N}=2 \mathrm{~K}\right), 8 \mathrm{~A}$ neutron scattering study $\underline{41}$ revealed that the antiferromagnetic structure is characterized by the wave vector $\mathbf{Q}=(0,0, \pi / c)$. Therefore, the normal state just above $T_{c}$ is invariant under the operation $\tau(\mathbf{c}) \mathcal{K}$ instead of $\mathcal{K}$, where $\tau(\mathbf{c})$ is a lattice translation along $z$-axis. This leaves valid all of the results of our discussion, since we consider translationally invariant superconducting states, and for the antiferromagnetic case, one still has $\varepsilon_{\mathbf{k}}=\varepsilon_{-\mathbf{k}}$. However, the presence of antiferromagnetic order can provide certain clues about possible superconducting state. It has been shown for zero SOC that the singlet superconducting state with gap function $\psi(\mathbf{k})$ is energetically favoured if $\psi(\mathbf{k}+\mathbf{Q})=-\psi(\mathbf{k})$ [See Ref. 7 and references therein]. Hence, the periodic basis functions in Table I, which depend on $k_{z}$, may be good candidates.

Even more exciting is the situation realized in UIr $\frac{9}{9}$ At ambient pressure it is ferromagnetic with Curie temperature of $46 \mathrm{~K}$. Superconductivity occurs at high pressures of 2.6-2.7 GPa. One possibility is that the phase transition lines from the normal paramagnetic to ferromagnetic state and from the normal paramagnetic to superconducting state meet at a quantum critical point. In that case superconducting states can again be classified with respect to the symmetry of time reversal invariant paramagnetic state. On the other hand, ferromagnetism can survive high pressures and coexist with the superconducting state. Then, time reversal symmetry is completely broken. Since the centre of symmetry is also missing, in general $\varepsilon_{\mathbf{k}} \neq \varepsilon_{-\mathbf{k}}$. Therefore, a zero-field analog of the inhomogeneous Larkin-Ovchinnikov-FuldeFerrel state, $\stackrel{42}{ }$ may be realized in this material. On the other hand, superconductivity exists in a relatively narrow pressure range. Hence, an alternative scenario may imply that $\varepsilon_{\mathbf{k}}$ and $\varepsilon_{-\mathbf{k}}$ are accidentally degenerate for a part of the Fermi surface. In either case, superconductivity in UIr deserves further investigation.

In conclusion, we have considered the symmetry properties of the gap function in superconductors with lifted spin degeneracy. We have shown that phase factors which appear with the gap function $\Delta(\mathbf{k})$ under point group operations and time reversal may be handled by the introduction of an even auxiliary function $\chi(\mathbf{k})$, which transforms according to the irreducible representations of the point group. This function defines the behaviour of the superconductor in terms of thermodynamic and tunneling properties.

Note added. The criticism of Ref. 10 presented in this Article is addressed in Ref. 43 . 


\section{Acknowledgments}

It is our pleasure to acknowledge very useful discussions with D.F. Agterberg and D.J. Singh. This work was supported by NSERC Canada.
* Electronic address: sergienko@ornl.gov Present address: Condensed Matter Sciences Division, Oak Ridge National Laboratory, Oak Ridge, Tennessee 37831, USA.

1 F. Steglich, J. Aarts, C.D. Bredl, W. Lieke, D. Meschede, W. Franz, H. Schäfer, Phys. Rev. Lett. 43, 1892 (1979).

2 G.E. Volovik and L.P. Gor'kov, JETP Lett. 39, 674 (1984).

3 P.W. Anderson, Phys. Rev. B 30, 4000 (1984).

${ }^{4}$ See, e. g., H. Jones, The Theory of Brillouin Zones and Electronic States in Crystals, 2nd rev. ed. (North-Holland, Amsterdam, 1975).

${ }^{5}$ G.E. Volovik and L.P. Gor'kov, Sov. Phys. JETP 61, 843 (1985).

${ }^{6}$ K. Ueda and T.M. Rice, Phys. Rev. B 31, 7114 (1985).

7 M. Sigrist and K. Ueda, Rev. Mod. Phys. 63, 239 (1991).

8 E. Bauer, G. Hilscher, H. Michor, Ch. Paul, E.W. Scheidt, A. Gribanov, Yu. Seropegin, H. Noël, M. Sigrist, and P. Rogl, Phys. Rev. Lett. 92, 027003 (2004).

9 T. Akazawa, H. Hidaka, T. Fujiwara, T.C. Kobayashi, E. Yamamoto, Y. Haga, R. Settai, and Y. Ōnuki, J. Phys.: Condens. Matter 16, L29 (2004).

10 K.V. Samokhin, E.S. Zijlstra, and S.K. Bose, Phys. Rev. B 69, 094514 (2004).

11 S.S. Saxena and P. Monthoux, Nature 427, 799 (2004).

12 M.C. Krupka, A.L. Giorgi, N.H. Krikorian, and E.G. Szklarz, J. Less-Common Met. 19, 113 (1969).

13 A.L. Giorgi, E.G. Szklarz, N.H. Krikorian, and M.C. Krupka, J. Less-Common Met. 22, 131 (1970).

14 G. Amano, S. Akutagawa, T. Muranaka, Y. Zenitani, and J. Akimitsu, J. Phys. Soc. Jpn. 73, 530 (2004).

15 D.J. Singh and I.I. Mazin, Phys. Rev. B 70, 052504 (2004).

16 This may not be the case for $\left(\mathrm{Y}_{1-x} \mathrm{Th}_{x}\right)_{2} \mathrm{C}_{3-y}$ and $\left(\mathrm{La}_{1-x} \mathrm{Th}_{x}\right)_{2} \mathrm{C}_{3-y}$, in which strong SOC is expected. As reported in Refs. 1213 , superconductivity with $T_{c}>10$ $\mathrm{K}$ survives the doping level up to $x=0.9$. Unfortunately, to the best of our knowledge, band structure calculations have not been reported for the Th doped compounds.

17 M. Hanawa, Y. Muraoka, T. Tayama, T. Sakakibara, J. Yamaura, and Z. Hiroi, Phys. Rev. Lett. 87, 187001 (2001).

18 H. Sakai, K. Yoshimura, H. Ohno, H. Kato, S. Kambe, R.E. Walstedt, T.D. Matsuda, Y. Haga and Y. Ōnuki, J. Phys.: Condens. Matter 13, L785 (2001).

19 J.-I. Yamaura and Z. Hiroi, J. Phys. Soc. Jpn. 71, 2598 (2002).

20 I.A. Sergienko and S.H. Curnoe, J. Phys. Soc. Jpn. 72, 1607 (2003).

${ }^{21}$ I.A. Sergienko, V. Keppens, M. McGuire, R. Jin, J. He, S.H. Curnoe, B.C. Sales, P. Blaha, D.J. Singh, K. Schwarz, and D. Mandrus, Phys. Rev. Lett. 92, 065501 (2004).

${ }^{22}$ H. Harima, J. Phys. Chem. Solids 63, 1035 (2002).

23 D.J. Singh, P. Blaha, K. Schwarz and J.O. Sofo, Phys. Rev. B 65, 155109 (2002).

${ }^{24}$ R. Eguchi, T. Yokoya, T. Baba, M. Hanawa, Z. Hiroi, N.
Kamakura, Y. Takata, H. Harima, and S. Shin, Phys. Rev. B 66, 012516 (2002).

25 M.D. Lumsden, S.R. Dunsiger, J.E. Sonier, R.I. Miller, R.F. Kiefl, R. Jin, J. He, D. Mandrus, S.T. Bramwell, and J.S. Gardner, Phys. Rev. Lett. 89, 147002 (2002).

26 O. Vyaselev, K. Arai, K. Kobayashi, J. Yamazaki, K. Kodama, M. Takigawa, M. Hanawa, and Z. Hiroi, Phys. Rev. Lett. 89, 017001 (2002).

27 A very recently reported ${ }^{195} \mathrm{Pt}-\mathrm{NMR}$ study of $\mathrm{CePt}_{3} \mathrm{Si}$ found the coherence peak below $T_{c}$, which indicates that the superconducting gap in $\mathrm{CePt}_{3} \mathrm{Si}$ is probably also nodeless [M. Yogi, Y. Kitaoka, S. Hashimoto, T. Yasuda, R. Settai, T.D. Matsuda, Y. Haga, Y. Ōnuki, P. Rogl, and E. Bauer, Phys. Rev. 93, 027003 (2004)].

28 L.D. Landau and E.M. Lifshitz, Quantum Mechanics (Non-Relativistic Theory), 3rd rev. ed., (Pergamon Press, Oxford, 1977).

29 V. Ambegaokar, in Superconductivity, Vol. II, edited by R.D. Parks (Marcel Dekker, New York, 1969).

30 The discussion of the transformation properties of the operators for spinless particles can be found in A. Messiah, Quantum Mechanics, Vol. II, (North Holland, Amsterdam, 1962), Chap. XXI. or J. D. Bjorken and S. D. Drell, Relativistic Quantum Fields, (McGraw-Hill, 1965), Chap. 15.

31 E.I. Blount, Phys. Rev. B 32, 2935 (1985).

32 L.P. Gor'kov, Sov. Phys. JETP 34, 506 (1958).

33 G.D. Mahan, Many-Particle Physics, 2nd ed. (Plenum Press, New York, 1990).

34 L.P. Gor'kov and E.I. Rashba, Phys. Rev. Lett. 87, 037004 (2001).

35 V. Ambegaokar and A. Baratoff, Phys. Rev. Lett. 10, 486 (1963).

36 E.I. Rashba, Sov. Phys. Solid State 2, 1109 (1960).

37 P.A. Frigeri, D.F. Agterberg, A. Koga, and M. Sigrist, Phys. Rev. Lett. 92, 097001 (2004).

38 See Ref. 37 and P.A. Frigeri, D.F. Agterberg, and M. Sigrist, New. J. Phys. 6, 115 (2004) for the discussion of $T_{c}$, spin susceptibility, etc.

39 P.W. Anderson, J. Phys. Chem. Solids 11, 26 (1959).

40 Time reversal of a fermion state $|\phi\rangle$ is defined as $|\phi\rangle^{*}$ in Ref. 39. Strictly speaking, it must be accompanied by changing the sign of one of the spinor components. Only then, the correct property $\mathcal{K}^{2}|\phi\rangle=-|\phi\rangle$ is obtained.

41 N. Metoki, K. Kaneko, T.D. Matsuda, A. Galatanu, T. Takeuchi, S. Hashimoto, T. Ueda, R. Settai, Y. Ōnuki, and N. Bernhoeft, J. Phys.: Condens. Matter 16, L207 (2004).

42 See for a recent review, R. Casalbuoni and G. Nardulli, Rev. Mod. Phys. 76, 263 (2004).

${ }^{43}$ K.V. Samokhin, E.S. Zijlstra, and S.K. Bose, Phys. Rev. B 70, 069902(E) (2004). 